\title{
Consideraciones sobre el uso de biofertilizantes como alternativa agro- biotecnológica sostenible para la seguridad alimentaria en México
}

\author{
Ismael Fernando Chávez-Díaz ${ }^{1}$ \\ Lily X. Zelaya Molina ${ }^{1}$ \\ Carlos Iván Cruz Cárdenas ${ }^{2}$ \\ Edith Rojas Anaya ${ }^{1}$ \\ Santiago Ruíz Ramírez ${ }^{3}$ \\ Sergio de los Santos Villalobos ${ }^{4 \S}$
}

${ }^{1}$ Laboratorio de Recursos Genéticos Microbianos. ${ }^{2}$ Laboratorio Agrícola Forestal de Semillas Ortodoxas, Centro Nacional de Recursos Genéticos- INIFAP. Boulevard de la Biodiversidad \# 400. Rancho las Cruces. CP. 47600. Tepatitlán de Morelos, Jalisco, México. (chavez.fernando@inifap.gob.mx; zelaya.lily@inifap.gob.mx; cruz.ivan@inifap.gob.mx; rojas.edith@inifap.gob.mx). ${ }^{3}$ Campo Experimental Centro Altos de Jalisco-INIFAP. Av. Biodiversidad \# 2470, Rancho las Cruces. CP. 47600. AP. 56. Tepatitlán de Morelos, Jalisco, México. (ruiz.santiago@inifap.gob.mx). ${ }^{4}$ Instituto Tecnológico de Sonora. 5 de febrero 818 Sur, Col. Centro, CP. 85000. Ciudad Obregón, Sonora, México.

${ }^{\S}$ Autor para correspondencia: sergio.delossantos@itson.edu.mx.

\section{Resumen}

Desde el Posclásico, sin saberlo, los agricultores mexicas aprovechaban la gran diversidad microbiana presente en el lecho de los lagos que utilizaban como sustrato en sus esquemas de producción agrícola. A través de los años, la agricultura mexicana evolucionó de forma exitosa a la intensificación del campo y logró incrementar significativamente la productividad agrícola. Sin embargo, la utilización incrementada de fertilizantes sintéticos como solución a los problemas de fertilidad del suelo condujo a elevados costos económicos, ambientales y sociales. El presente ensayo muestra una reflexión crítica sobre la situación actual en el uso de los biofertilizantes en el campo mexicano y consideraciones futuras para garantizar su uso exitoso, y así contribuir a la seguridad alimentaria nacional de forma sostenible.

Palabras clave: bacterias promotoras de crecimiento vegetal, sostenibilidad, suelo.

Recibido: junio de 2020

Aceptado: julio de 2020 


\section{El desarrollo de la agricultura en México y a nivel mundial}

Los registros de la actividad agrícola en México datan del Posclásico, en donde los mexicas habitantes de la zona lacustre de Xochimilco y Chalco (1200 a 1350 DC)- diseñaron un sistema de hidroponía rudimentario llamado 'Chinampas', en el que se utilizaba el lecho fangoso del lago (con abundante diversidad microbiana) para establecer los cultivos. Durante este periodo, el objetivo de la agricultura era abastecer con alimentos a las ciudades prehispánicas en crecimiento (BastidaTapia, 2017).

Con la llegada de los europeos, en el México colonial (a partir de 1520), se incorporaron a la agricultura utensilios metálicos de labranza, animales de tiro (bovinos y bueyes) y de transporte (caballos, asnos y mulas) y se estableció el monocultivo incorporando cultivos como el trigo, la caña de azúcar, el tabaco, el algodón y el café. El sistema colonial se sostuvo; a partir, de la explotación de los pueblos indígenas y de los ecosistemas, rompiendo el equilibrio agroecológico que mantenían los pueblos prehispánicos e iniciando con el agotamiento de los suelos mexicanos (WRM, 2004; Cruz-León et al., 2010).

Posteriormente, la agricultura mexicana puede describirse a grandes rasgos en tres etapas: i) etapa de crecimiento (1940-1957), en donde la agricultura se impulsó como principal actividad económica logrando representar hasta el 19\% del producto interno bruto (PIB) nacional; ii) etapa de desarrollo para el país (1958-1981), en donde el sector agrícola fue un componente clave para el abastecimiento de la industrialización de México; y iii) etapa actual (de 1982 a la fecha), en la cual la agricultura se ve afectada por la crisis de 1982, la deuda externa y la caída del tipo de cambio, pero al mismo tiempo fue favorecida por la apertura de la economía del Tratado de Libre Comercio (TLCAN) que diversificó los productos e incrementó el uso de tecnología agrícola (Gómez-Oliver, 1995).

Por otra parte, el crecimiento acelerado de la población a nivel mundial a mediados del siglo pasado demandó el incremento continuo de la producción y calidad de productos agroalimentarios (Huerta et al., 2018). Lo anterior, impulsó al Fitopatólogo Norman E. Borlaug, considerado 'el padre de la agricultura moderna', a emprender un proyecto innovador en 1944 que consistió en aumentar la productividad agrícola al incorporar avances tecnológicos para potenciar el rendimiento de los cultivos, a lo cual se le denominó Revolución Verde (Cerutti, 2019).

La Revolución Verde se enfocó en el monocultivo de variedades mejoradas; por ejemplo, entre los rasgos agronómicos para el cultivo de trigo destacaron i) el considerable acortamiento de los tallos, característica importante que favorece su rendimiento y evita el acame de la planta por el peso incrementado de los granos; y ii) el aumento de la adaptabilidad a la latitud, elevación, y otros factores ambientales. Lo anterior, incrementó el rendimiento del trigo de $2 \mathrm{t} \mathrm{ha}^{-1}$ a $8 \mathrm{t} \mathrm{ha}^{-1}$ (Diéguez et al., 2010).

Por otra parte, las prácticas agrícolas utilizadas para dichas variedades mejoradas se basaron en el uso riego, mecanización y la aplicación de pesticidas y fertilizantes sintéticos y la aplicación de fertilizante nitrogenado $(\mathrm{N})$ a nivel mundial aumentó de $32 \mathrm{Tg} \mathrm{N}$ (millones de toneladas métricas) en 1970 a aproximadamente $80 \mathrm{Tg}$ en 1990 (McCullough y Matson, 2016). 
De esta manera, la Revolución Verde fue un éxito al impulsar la producción de los principales cereales a nivel mundial (trigo, arroz y maíz, entre otros); sin embargo, su impacto a gran escala ha sido discutido. La aplicación de altas dosis de fertilizantes sintéticos a partir de los años 60's (hasta $250 \mathrm{~kg} \mathrm{ha}^{-1}$ en la actualidad) (McCullough y Matson, 2016) provocó que entre 1960 y 2000, los rendimientos agrícolas aumentaran 208\% para el trigo, 109\% para el arroz, $157 \%$ para el maíz, $78 \%$ para la papa y $36 \%$ para la yuca en los países en desarrollo, generando grandes ingresos económicos y alimenticios a nivel mundial (Lobell et al., 2005).

Sin embargo, en varios países en desarrollo incluyendo a México, las variedades de alto rendimiento y las técnicas de siembra intensivas fueron adquiridas casi en su totalidad por grandes agricultores establecidos comercialmente, a diferencia de los pequeños agricultores rurales que no contaban con las mismas oportunidades (Harwood, 2009), potenciando la polarización económica, así como la desvalorización de las técnicas tradicionales y del medio rural (de Grammont, 2010) además, el uso excesivo de fertilizantes sintéticos generó un impacto negativo a nivel ambiental, poniendo en riesgo la salud de productores, consumidores y los recursos productivos, genéticos y la biodiversidad (Naylor et al., 2001).

\section{Uso de fertilizantes sintéticos y su impacto en la actividad agrícola en México}

La aplicación de fertilizantes sintéticos en la agricultura incrementa en gran medida el rendimiento de diversos cultivos, por lo cual su uso aumentó 27.1\% en América Latina y el Caribe durante el periodo 2006-2017 (Reyes y Cortes, 2017). A pesar de estos beneficios, diversos autores reportan que el uso excesivo e inapropiado de fertilizante sintéticos ocasiona graves problemas ambientales y ecológicos (Snyder, 2009).

El potencial de contaminación de un fertilizante sintético se encuentra estrechamente relacionado con su uso eficiente por el cultivo, lo cual varía en función de factores como el tipo de fertilizante y su presentación, el momento y la manera de suministrarlo al cultivo y la tecnología industrial de fabricación (García, 2009). De esta manera, el uso inadecuado de fertilizantes sintéticos impacta directamente en la actividad agrícola generando elevados costos económicos, deterioro ambiental y segregación social.

Impacto económico. Tomando el cultivo de maíz como ejemplo, producir una hectárea bajo un esquema agrícola convencional en el periodo otoño-invierno 2011 a primavera-verano 2012 tuvo un costo entre \$9 763.00 y $\$ 24033.00$ pesos mexicanos, destinando entre $23.3 \%$ y $25.9 \%$ a la fertilización y control de plagas y malezas; Sin embargo, durante el periodo 2019 a 2020 el costo incremento de $\$ 15112.00$ hasta $\$ 42007.00$ pesos mexicanos, destinando de $31 \%$ a $35 \%$ de este costo a la fertilización y el control fitosanitario (FIRA, 2020). Aun cuando el rango de costos es amplio [debido al sistema de producción (riego o de temporal), las variables agroclimáticas, rendimiento esperado, grado de tecnificación, entre otros], es notable el incremento en el porcentaje de éste destinado a la fertilización y control fitosanitario con productos de origen sintético.

Asimismo, este costo se ve impactado por el incremento de los precios de dichos insumos sintéticos, la fluctuación de los precios del producto en el mercado y en su caso, por la variación de los servicios financieros a los que recurre el productor. Este modelo se repite en los diversos cultivos producidos en México y afecta directamente al margen de ganancia del productor y al nivel adquisitivo del consumidor. 
Impacto ambiental. Éste es causado por el grado de perturbación que las prácticas agrícolas causan al ambiente, principalmente sobre la calidad del suelo, el agua, el aire, y la biodiversidad, así como en la salud de personas, animales y plantas de una región determinada (Balmford et al., 2018). Los modelos actuales de producción basan el manejo de los cultivos en el uso excesivo de fertilizantes sintéticos para la nutrición de los cultivos y de compuestos tóxicos para el control de plagas, enfermedades y malezas (FAO, 2002). La alta concentración de estos compuestos en suelo, agua y aire, genera un desbalance en los ciclos biogeoquímicos y las cadenas tróficas de las zonas agrícolas, teniendo como efecto la disminución de la capacidad productiva, la dificultad en el control de plagas, enfermedades y malezas que han generado resistencia (Mandal et al., 2020).

Impacto social. El modelo agrícola actual, adoptado desde 1958, también ha tenido una secuela social, ya que ha generado una polarización económica-social acompañada por segregación y discriminación hacia el sector rural (de Grammont, 2010). Los grandes productores y empresas trasnacionales invierten en la agricultura tecnificada, la cual tiene altos costos económicosambientales y los pequeños productores al no poder competir ante estas condiciones rentan sus tierras o terminan trabajándolas como empleados agrícolas recibiendo salarios bajos que apenas permiten cubrir sus necesidades de alimentación (López-Feldman y Herández-Cortés, 2016).

Durante las últimas décadas, el éxito de los sistemas agrícolas convencionales ha sido importante y significativo; sin embargo, éstos han ocasionado la pérdida de la diversidad biológica, disminución de recursos forestales, erosión del suelo, cambios climáticos, entre otros. En este contexto, los retos actuales de la producción agrícola nacional se centran en: i) generar cambios en las políticas agrarias que promuevan la disgregación de la polarización social y económica del campo (que ha prevalecido en la agricultura mexicana), potenciando el sector rural y conservándolo como un instrumento para promover la seguridad alimentaria en el país; y ii) migrar de forma paulatina y progresiva hacia métodos de producción agrícola costo-efectivos y con una visión holística que permita la recuperación y preservación de suelos, agua, recursos genéticos y que no signifiquen un riesgo para la salud ambiental, del productor o del consumidor.

\section{La microbiología y su relación con la agricultura: el caso de los biofertilizantes}

Las plantas están en constante interacción con su entorno y principalmente se encuentran relacionadas con el suelo, el cual es un amplio y complejo ecosistema en el que también habitan grandes poblaciones microbianas. Los suelos con mayores contenidos de materia orgánica (>2\%) contienen una incrementada población y diversidad de microorganismos, conformadas principalmente por bacterias, actinomicetos, hongos y algas (Jacoby et al., 2017), cuya actividad está fuertemente relacionada con la fertilidad y estabilidad del recurso edáfico (de los SantosVillalobos et al., 2018).

De esta manera, el suelo puede llegar a albergar una población de $10^{8}$ hasta $10^{9}$ células bacterianas por gramo; sin embargo, si éste es sometido a cualquier tipo de estrés (escasez de agua y nutrientes, salinidad del suelo, contaminaciones por metales pesados, entre otros) consecuencia de las prácticas agrícolas, la población puede disminuir hasta $10^{4}$ células bacterianas por gramo de suelo (Valenzuela-Aragon et al., 2019). Así, la diversidad microbiana en los suelos se estima en más de $10^{5}$ especies, las cuales están involucradas en i) el ciclaje de nutrientes; ii) la descomposición de la materia orgánica; iii) la fotosíntesis; iv) la biorremediación; y v) el control de enfermedades de las plantas, entre otros (de los Santos-Villalobos et al., 2018). 
La rizósfera, porción de suelo en la cual las raíces de las plantas tienen influencia a través de sus exudados, posee la mayor población y diversidad de microorganismos, los cuales compiten por espacio y nutrientes (Jacoby et al., 2017). Estas interacciones microbianas impactan directamente las relaciones suelo-planta-microorganismos-ambiente y repercuten de forma positiva o negativa en el crecimiento y en el desarrollo de los cultivos agrícolas (Cano, 2011).

Entre los microorganismos que habitan en la rizósfera se distinguen aquellos con la capacidad de favorecer el desarrollo y el rendimiento de los cultivos; a través, de mecanismos directos e indirectos (Figura 1), los cuales son llamados microorganismos promotores de crecimiento vegetal (MPCV). Los mecanismos directos mejoran el estado nutricional de la planta al incrementar el volumen de exploración y funcionalidad de las raíces, la captación de agua, la disponibilidad y absorción de nutrientes y la fisiología de toda la planta (Kumar et al., 2015). Esto se lleva a cabo mediante la producción de reguladores de crecimiento, ácidos orgánicos, enzimas, metalóforos, vitaminas y otros metabolitos secundarios que impactan directamente en el crecimiento de la planta (Grageda-Cabrera et al., 2012; Moreno-Reséndez et al., 2018).

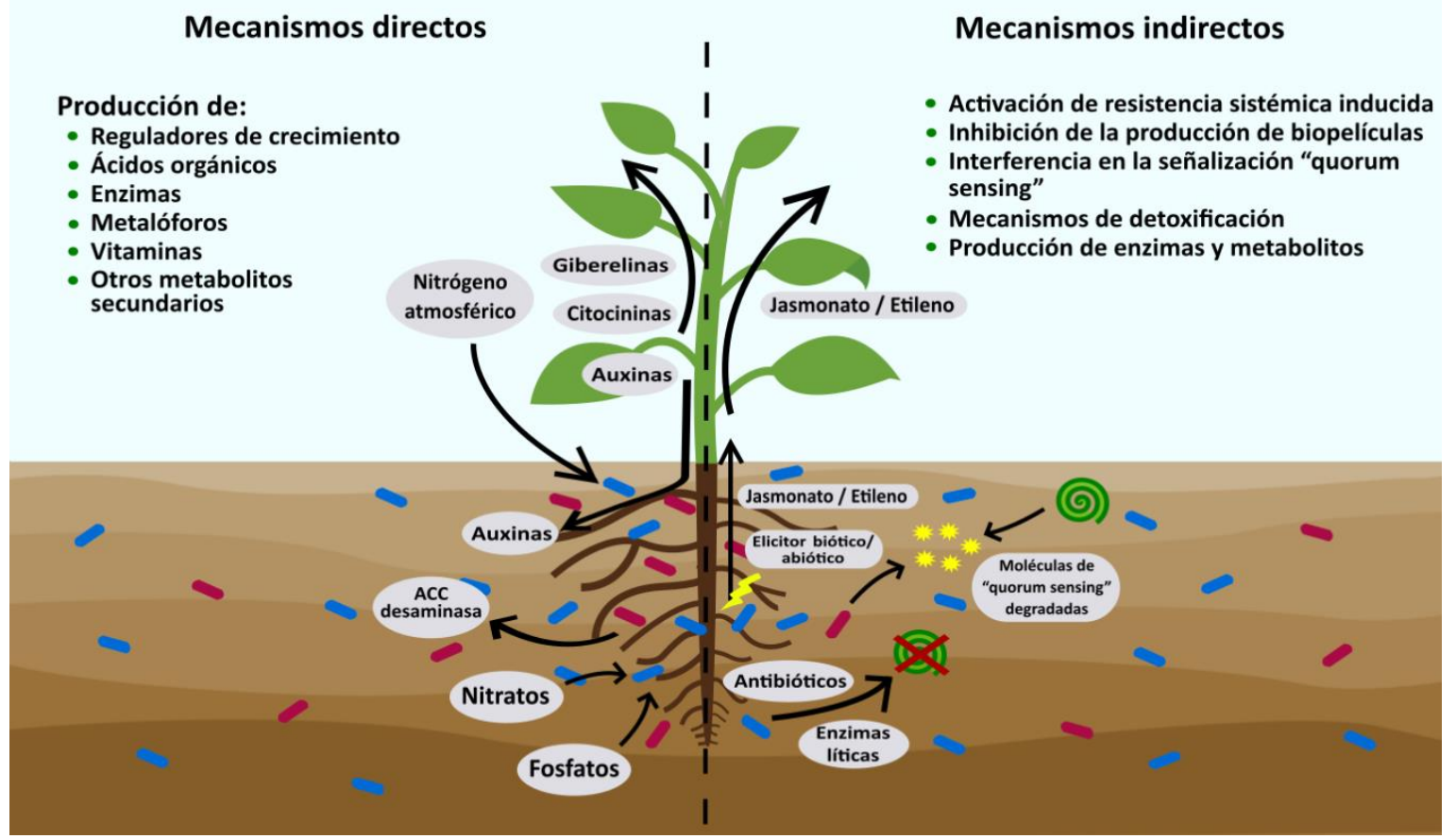

Figura 1. Mecanismos directos e indirectos presentes en microorganismos promotores del crecimiento vegetal (MPCV).

Por otra parte, los mecanismos indirectos están involucrados en la protección contra el estrés ocasionado por factores abióticos y bióticos, entre los que destacan la inducción de resistencia a condiciones ambientales adversas y a fitopatógenos. Este último involucra la activación de la resistencia sistémica inducida, inhibición de producción de biopelículas, interferencia en la señalización 'quorum sensing', activación de mecanismos de detoxificación de factores de virulencia, y la producción de enzimas/metabolitos involucrados en funciones especializadas (Moreno-Reséndez et al., 2018; Villarreal-Delgado et al., 2018). 
La anterior ha propiciado el uso de MPCV como ingredientes activos de los biofertilizantes, los cuales son bio-formulados que contienen microorganismos vivos, al ser aplicados de manera foliar, en riego o al suelo, promueven el desarrollo de las plantas; a través, de los mecanismos directos e indirectos anteriormente mencionados (Santoyo et al., 2019). Entre los géneros bacterianos empleados en la producción de biofertilizantes destacan Rhizobium, Bacillus y Pseudomonas.

Las especies de Rhizobium, además de fijar nitrógeno atmosférico, incrementan el crecimiento, rendimiento y número de nódulos por raíz y movilizan fósforo (Saharan y Nehra, 2011). Las investigaciones recientes en este género bacteriano como promotor del crecimiento vegetal se enfocan en analizar su efecto sobre i) la estructura de las comunidades microbianas asociadas a la raíz (Jha et al., 2020); ii) desarrollar biofertlizantes para múltiples cultivos de leguminosas (Passricha et al., 2020); iii) introducir células de Rhizobium dentro de semillas mediante tecnología de vació para evitar pérdidas del inóculo (Lekatompessy et al., 2020); y iv) evaluar el efecto de la co-inoculación de Rhizobium y esporas de endomicorrizas (Kiuk et al., 2019).

Bacillus es el género más abundante en la rizósfera, cepas de B. subtilis, B. megaterium, $B$. mucilaginosus, B. pumilus y B. licheniformis son las especies más estudiadas por su capacidad de colonización, solubilización de potasio y fósforo, aumento del desarrollo, longitud y materia seca de la raíz, y rendimiento de las plantas (Bhattacharyya y Jha, 2012; de los Santos-Villalobos et al., 2019; Villa-Rodríguez et al., 2019). Actualmente, los estudios sobre este género bacteriano se enfocan en i) nuevas estrategias para la producción de sus esporas en cultivo sólido o líquido (Hindersah et al., 2020); ii) evaluar el efecto de cepas de Bacillus sobre el contenido de compuestos fenólicos en las plantas (Jiménez-Gómez et al, 2020); iii) determinar el tiempo de cosecha para maximizar el contenido nutricional de frutos biofertilizados con cepas de Bacillus (Cisternas-Jamet et al., 2020); y iv) analizar el nivel de expresión del potencial genómico de cepas de Bacillus durante la interacción planta-rizósfera (Borriss, 2020).

El género Pseudomonas es ubicuo en el suelo, siendo las cepas más efectivas de este género las pertenecientes a la especie Pseudomonas fluorescentes, las cuales ayudan en el mantenimiento de la salud del suelo; a través, de una gran diversidad metabólica y funcional (Lugtenberg y Dekkers, 1999). Actualmente, las investigaciones sobre la generación de biofertilizantes conteniendo cepas de este género bacteriano se enfocan en i) analizar el potencial de cepas de Pseudomonas modificadas genéticamente sobre el rendimiento de cultivos agrícolas (Wang et al., 2020); ii) identificar cepas promotoras de crecimiento vegetal con la capacidad de biorremediar suelos contaminados con metales pesados (Khashei et al., 2020); iii) identificar cepas con la capacidad de incrementar la tolerancia a salinidad en cultivos (Lami et al., 2020); y iv) evaluar la participación de genes específicos de algunas cepas en la promoción de crecimiento vegetal (Tahir et al., 2020).

Por otra parte, entre los hongos promotores del crecimiento vegetal, las cepas más estudiadas pertenecen al género Glomus, las cuales han sido reportadas como agentes mitigadores de los efectos inducidos por el estrés hídrico en las plantas (Mota et al., 2020) e incrementar el crecimiento vegetal por acción sinérgica al co-inocularse con bacterias promotoras del crecimiento vegetal (Nadeem et al., 2014). Además, diversas cepas de Trichoderma se han estudiado por su i) potencial de antagonismo y micoparasitismo contra fitopatógenos; ii) capacidad de mejorar el crecimiento vegetal en condiciones de estrés abiótico (Hermosa et al., 2012); iii) capacidad de incrementar el contenido de pigmentos en las plantas (Metwally y Al-Amri, 2020); y iv) habilidad de potenciar la microbiota y actividad enzimática del suelo (Zhang et al., 2020). 


\section{Consideraciones en el manejo agrícola y factores que limitan la eficiencia de los biofertilizantes}

Actualmente, los biofertilizantes se emplean exitosamente en muchos países desarrollados, mientras que en los países en desarrollo su uso y aprobación -por el sector agrícola- está limitado por diversos factores, ie. el conocimiento sobre su manejo adecuado (Grageda-Cabrera et al., 2012). De esta manera, es importante conocer y divulgar información científica sobre el correcto uso de los biofertilizantes, sus asociaciones con las plantas y las condiciones del agrosistema y de manejo que afectan dichas interacciones.

Los métodos recomendados para la aplicación de biofertilizantes son: inoculación de semillas, inoculación en plántulas por inmersión, aplicación en el sistema de riego y aplicación al suelo. Además, las principales recomendaciones para el uso exitoso de los biofertilizantes se mencionan a continuación (Figura 2).

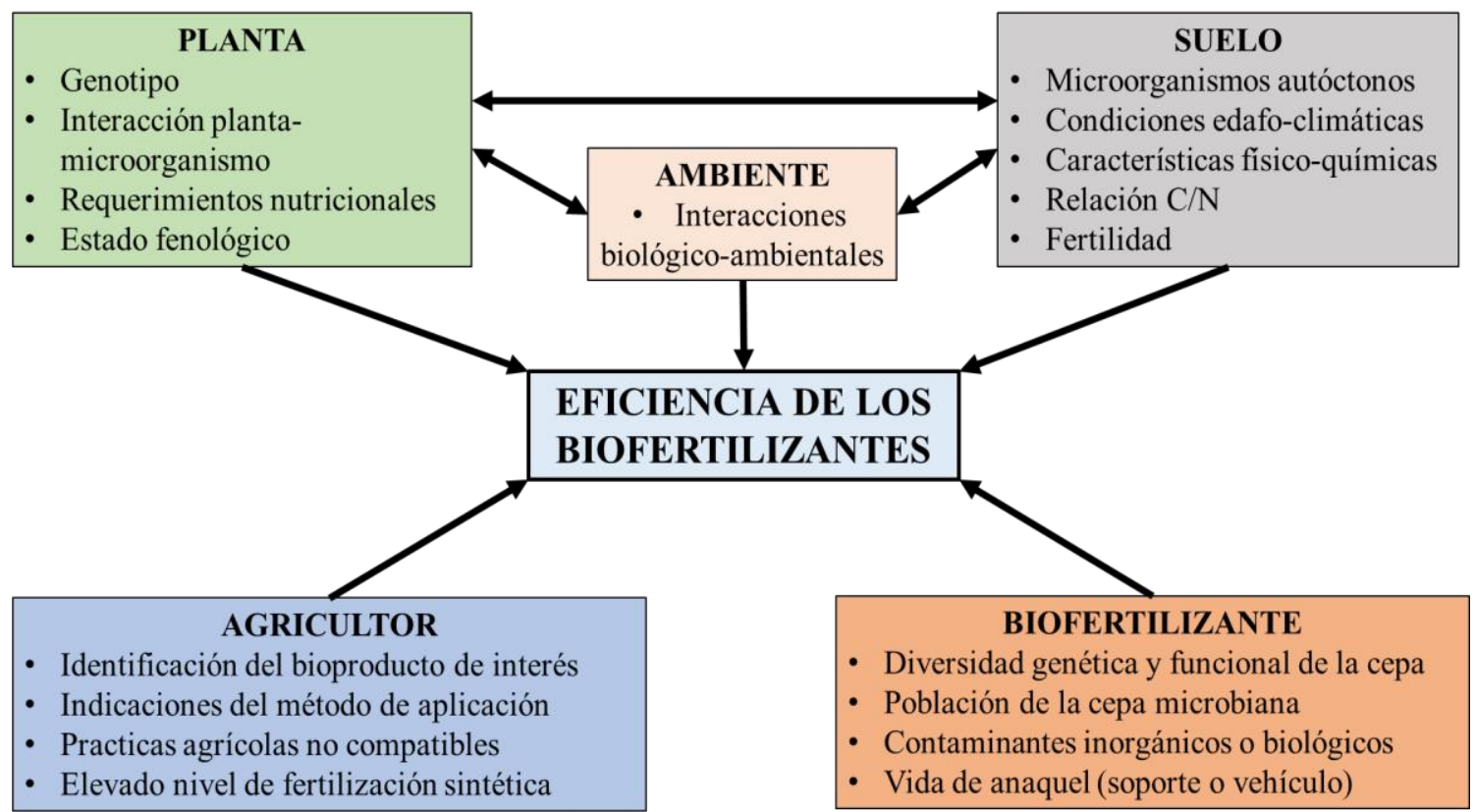

Figura 2. Consideraciones para el uso exitoso de biofertilizantes en el sector agrícola.

A) el biofertilizante debe contener una apropiada población viva de la cepa reportada como ingrediente activo, y estar libre de microorganismos contaminantes (Sanjuán-Pinilla y MorenoSarmiento, 2010). B) el biofertilizante debe ser seleccionado con base en el ingrediente activo (cepa microbiana), el cultivo de interés, las condiciones edafo-climáticas y prácticas agrícolas empleadas; además, dicho bioproducto debe ser empleado antes de la fecha de caducidad (Moreno-Reséndez et al., 2018); C) la aplicación en campo de los biofertilizantes debe hacerse de acuerdo a lo establecido por el proveedor. Por ejemplo, el uso de compuestos adherentes es determinante para el tratamiento de semillas con biofertilizantes (Bojórques et al., 2010); y D). Los biofertilizantes deben almacenarse en un lugar fresco y seco, lejos de la luz solar directa y el calor y emplearse en combinaciones correctas con agroquímicos (Sanjuán-Pinilla y Moreno-Sarmiento, 2010). 
El éxito de la aplicación de los biofertilizantes también depende del soporte o acarreador, el cual determina la vida útil del producto y la persistencia de sus microorganismos durante la fenología del cultivo o en la etapa de interés para su efecto benéfico (Ansari et al., 2015). Así, la población de células viables inoculadas es de gran importancia para la promoción del crecimiento esperada en el cultivo, ya que un número excesivo o limitado de éstas puede dificultar la germinación de la semilla o el crecimiento de la planta, respectivamente (Boddey y Dobereiner, 1995).

Por otra parte, el conocimiento de los requerimientos nutricionales y ambientales de los microorganismos contenidos en los biofertilizantes es determinante para su efectividad, así como su capacidad de colonización de la planta, adaptación al suelo e interacción con los microorganismos nativos (Khalid et al., 2004; Grageda-Cabrera et al., 2012). La disponibilidad de nutrientes, el pH y la salinidad determinan la sobrevivencia de los microorganismos en el suelo, la escasez o exceso de algún compuesto químico puede disminuir rápidamente la población microbiana inoculada.

El contenido de la materia orgánica y nitrógeno (relación $\mathrm{C}$ : $\mathrm{N}$ ) en el suelo afectan significativamente las funciones de promoción del crecimiento de vegetal de las cepas contenidas en los biofertilizantes (Dobbelaere et al., 2001; Grageda-Cabrera et al., 2012). Además, se ha reportado que a menor fertilidad del suelo, mayor es la estimulación del crecimiento de las plantas por los biofertilizantes, ya que movilizan los elementos no disponibles y recalcitrantes (De Freitas and Germida, 1990) y otros estudios evidencian que el elevado nivel de fertilización sintética al cultivo inhibe o disminuye la efectividad de estos bioproductos. De esta manera, en la actualidad existen numerosos estudios enfocados en conocer el equilibrio económico, ambiental y funcional, para el uso de biofertilizantes en combinación con niveles reducidos de fertilización sintética en los cultivos agrícolas (Spolaor et al., 2016). Así, el uso de biofertilizantes impactará positivamente la seguridad alimentaria actual y futura, y la mitigación de los efectos negativos a nivel económico, social y ambiental generados por los sistemas de producción agrícola convencionales.

\section{Casos de éxito en el uso de biofertilizantes en México}

Los biofertilizantes han sido ampliamente aceptados internacionalmente, ya que han demostrado diversas ventajas en el campo. En México, diversas investigaciones se han desarrollado sobre el desarrollo, innovación y validación de los biofertilizantes. Por ejemplo, un biofertilizante que ha mostrado impactos positivos y significativos es el desarrollado por Trujillo-Roldan et al. (2013). Los autores lograron un incremento de $70 \%$ en el peso de la biomasa aérea en el maíz y 95\% en el incremento de biomasa en las mazorcas por la aplicación de Azospirillum brasilense, en comparación con un fertilizante sintético. Parra-Cota et al. (2014) reportaron que mediante la inoculación de dos especies de Burkholderia (B. ambifaria Mex5 y B. caribensis XV) se incrementó el rendimiento hasta $155.4 \%$ y $41.4 \%$ en el amaranto (comparado con el tratamiento no inoculado), respectivamente, en condiciones de suelos arenosos. De forma similar, RojasPadilla et al. (2020) reportaron que la inoculación del trigo por un consorcio bacteriano compuesto por B. megaterium TRQ8 + B. paralicheniformis TRQ65 mostró los mayores incrementos significativos ( $v s$ el tratamiento no inoculado) en la longitud aérea y radical de 6 y $10 \%$, respectivamente, mientras que la biomasa seca aérea y radical aumentó $60 \%$ y 82\%, respectivamente. 
Asimismo, los hongos micorrícicos han demostrado ser altamente eficiente para establecer asociaciones con las plantas. Por ejemplo, Aguirre-Medina et al. (2004) reportaron que la coinoculación de algunos hongos micorrícicos (Rhizophagus intraradices) y cepas de Rhizobium y Azospirillum promovieron el desarrollo vegetal de cultivos anuales y perennes. Recientemente, la inoculación de hongos micorrícicos al cultivo de trigo condujo a un incremento de la producción de grano de hasta $1291 \mathrm{~kg} \mathrm{ha}^{-1}$ (Grageda-Cabrera et al., 2011).

Por otra parte, Hipolito-Romero y et al. (2017) reportaron que la co-inoculación de dos cepas fijadoras de nitrógeno (Azospirillum brasilense UAP-151 y UAP-154) y dos cepas solubilizadoras de fosforo (Chromobacterium violaceum BUAP 35 y Acinetobacter calcoaceticus BUAP40) mostró efectos benéficos sobre parámetros agronómicos de las plantas, incrementando la altura (49\%), el diámetro (127\%), el número de ramas (300\%) y el número de hojas (500\%), en comparación con las plantas tratadas con fertilizantes sintéticos.

Aunque aquí se presentan sólo algunos casos de éxito en el uso de biofertilizantes en México, la aceptación y aplicación de esta agro-biotecnología sin duda incrementará, debido a su efectividad, bajo costo, nulos impactos negativos al ambiente, y al ser una meta del programa nacional de biofertilizantes, para promover una agricultura sostenible. Así, el conocimiento de los beneficios del uso de los biofertilizantes sin duda permitirá tomar decisiones enfocadas a la producción de alimentos de forma sostenible en México.

\section{Conclusiones}

En la actualidad, el desarrollo de biofertilizantes debe considerar diversos aspectos, entre los cuales destacan: i) la selección y evaluación de cepas nativas efectivas para obtener rendimientos óptimos y sostenibles, potenciar la interacción planta-microorganismo, cultivos objetivo, respuesta a factores ambientales y preservación del recurso microbianos nativos; ii) la investigación sobre formulaciones mejoradas de inoculantes, vida útil, beneficios residuales, persistencia y adaptaciones al estrés de cepas microbianas; iii) el monitoreo del control de calidad en las etapas de producción, distribución, aplicación en campo mediante el cumplimiento estricto de los lineamientos y regulaciones; iv) la integración de los biofertilizantes a otras prácticas agroecológicas adaptadas a diferentes sistemas de cultivo para lograr una agricultura sostenible; v) el desarrollo de políticas y estrategias que permitan hacer llegar los biofertilizantes a grupos de agricultores, instituciones de investigación y aprendizaje, organizaciones privadas y públicas; y vi) establecer redes que involucren a instituciones locales, sector privado y organizaciones de investigación para desarrollar modelos efectivos para la producción de biofertilizantes con microorganismos nativos de las regiones en donde se aplicarán. Lo anterior conducirá al uso eficiente de biofertilizantes como una estrategia sostenible para alcanzar la seguridad alimentaria actual y futura en México.

\section{Agradecimientos}

El financiamiento otorgado por el Instituto Tecnológico de Sonora al Proyecto PROFAPI 2020. 0013 'Bacillus sp. TSO9: afiliación taxonómica a nivel del genoma e identificación de genes asociados a la promoción del crecimiento en el trigo' (S. de los Santos Villalobos). Los autores(as) agradecen las valiosas contribuciones de Jairo Eder Guerra Camacho y Claudia Fernanda Valenzuela Parra al presente manuscrito. 


\section{Literatura citada}

Aguirre-Medina, J. F.; Irizar-Garza, M. B.; Durán-Prado, A.; Grajeda-Cabrera, O. A.; Peña-Río, M. A. y Loredo-Osti B. A. 2004. Los biofertilizantes microbianos: alternativa para la agricultura en México. In: Andrade, A. y Gutiérrez, G. B. (Eds.). Manual de microbiología. $2^{\mathrm{a}}$. (Ed.). México DF. Ed Trillas. 86-91 pp.

Ansari, M. F.; Tipre, D. R. and Dave, S. R. 2015. Efficiency evaluation of commercial liquid biofertilizers for growth of Cicer aeritinum (chickpea) in pot and field study. Biocatalysis Agric. Biotechnol. 4(1):17-24.

Balmford, A.; Amano, T.; Bartlett, H.; Chadwick, D.; Collins, A.; Edwards, D.; Fiel, R.; Garnsworthy, P.; Green, R.; Smith, P.; Waters, H.; Whitmore, A.; Broom, D. M.; Chara, J.; Finch, T.; Garnett, E.; Galthore-Hardy, A.; Hernández-Medrano, J.; Herrero, M.; Hua, F.; Latawiec, A.; Misselbrook, T.; Phalan, B.; Simmons, B. I.; Takahashi, T.; Vause, J.; Ermgassen, E. and Eisner, R. 2018. The environmental costs and benefits of high-yield farming. 1:477-485. https://doi.org/10.1038/s41893-018-0138-5.

Bastida-Tapia, A. 2017. Evolución y situación actual de la agricultura protegida en México. In: Sexto congreso Internacional de Investigación en Ciencias Básicas y Agronómicas. 281-294 pp.

Bhattacharyya, P. N. and Jha, D. K. 2012. Plant growth-promoting rhizobacteria (PGPR): emergence in agriculture. World J. Microbiol. Biotechnol. 28(4):1327-1350. https://doi.org/10.1007/s11274-011-0979-9.

Boddey, R. M. and Dobereiner, J. 1995. Nitrogen fixation associated with grasses and cereals: recent progress and perspectives for the future. $I n$ : nitrogen economy in tropical soils. Fertilizer Research. Dordrecht. 42: 241-250. https://doi.org/10.1007/BF00750518.

Borriss, R. 2020. Phytostimulation and biocontrol by the plant-associated Bacillus amyloliquefaciens FZB42: an update. In Phyto-Microbiome in Stress Regulation Springer, Singapore. 1-20 pp. https://doi.org/10.1007/978-3-319-44409-3_8.

Cano, M. A. 2011. Interacción de microorganismos benéficos en plantas: micorrizas, Trichoderma spp. y Pseudomonas spp. Revista U.D.C.A Actualidad \& Divulgación Científica. Colombia. 14(2):15-31. https://doi.org/10.31910/rudca.v14.n2.2011.771.

Cerutti, M. 2019. Trigo y revolución verde en el noroeste de México (1930-1970). Mundo agrario, 20(43):103-124. https://doi.org/10.24215/15155994e103.

Cisternas-Jamet, J.; Salvatierra-Martínez, R.; Vega-Gálvez, A.; Stoll, A.; Uribe, E. and Goñi, M. G. 2020. Biochemical composition as a function of fruit maturity stage of bell pepper (Capsicum annum) inoculated with Bacillus amyloliquefaciens. Sci. Hortic. 263:109-107. https://doi.org/10.1016/j.scienta.2019.109107.

Cruz-León, A.; Martínez-Saldaña, T. and Damián-Huato, M. A. 2010. Las mulas olvidadas por la historia agrícola colonial: una restitución. Agric. Soc. Des. 7(3):221-228.

De Freitas, J. R. and Germida, J. J. 1990. Plant growth promoting rhizobacteria for winter wheat. Canadian J. Microbiol. 36(4):265-272. https://doi.org/10.1139/m90-046.

De Grammont, H. C. 2010. Evolution of agricultural production in mexican countryside: Concentratio of production, poverty a pluriactivity. Andamios: Rev. Investig. Soc. 7(13):85-117. 
De los Santos-Villalobos, S.; Robles, R. I.; Parra-Cota, F. I.; Larsen, J.; Lozano, P. and Tiedje, J. M. 2019. Bacillus cabrialesii sp. nov., an endophytic plant growth promoting bacterium isolated from wheat (Triticum turgidum subsp. durum) in the Yaqui Valley, Mexico. Inter. J. Systematic Evol. Microbiol. 69(12):3939-3945. https://doi.org/10.1099/ijsem.0.003711.

De los Santos Villalobos, S.; Parra-Cota, FI.; Herrera Sepúlveda, A.; Valenzuela-Aragon, B. y Estrada Mora, J. C. 2018. Colmena: colección de microorganismos edáficos y endófitos nativos, para contribuir a la seguridad alimentaria nacional. Rev. Mex. Cienc. Agríc. 9(1):191-202. http://dx.doi.org/10.29312/remexca.v9i1.858.

Diéguez, T.; Cruz-Falcón, E.; Norzagaray-Campos, A.; Beltrán-Morales, M.; Murillo-Amador, L.; Beltrán-Morales, B.; Félix, A.; García-Hernández, J. L. y Valdez-Cepeda, R. 2010. Agotamiento hidro-agrícola a partir de la Revolución Verde: extracción de agua y gestión de la tecnología de riego en Baja California Sur, México. Estudios sociales (Hermosillo, Son.). México. 18(36):177-201.

Dobbelaere, S.; Croonenborghs, A.; Thys, A.; Ptacek, D.; Vanderleyden, J.; Dutto, P.; LabanderaGonzalez, C.; Caballero-Mellado, J.; Aguirre, J. F.; Kapulnik, Y.; Brener, S.; Burdman, S.; Kadouri, D.; Sarig, S. and Okon, Y. 2001. Responses of agronomically important crops to inoculation with Azospirillum. Australian J. Plant Physiol. 28(9):871-879. https://doi.org/10.1071/PP01074.

FAO. 2002. Agricultura mundial hacia los años 2015/2030. Food and Agriculture Organization. ISBN 92-5-304761-5.

FIRA. 2020. Fideicomisos Instituidos en Relación con la Agricultura. Sistema de costos agrícolas: resumen de costos. 1-5 pp.

García, M. J. P. 2009. Manejo eficiente de nutrientes en el cultivo de maíz. Ministerio de Agricultura y Desarrollo Rural. Colombia. 59 p.

Gómez-Oliver, L. 1995. El papel de la agricultura en el desarrollo de México. Oficina Regional de la FAO para América Latina y el Caribe (Ed.). Santiago de Chile. 1-52 pp.

Grageda-Cabrera, O. A.; González-Figueroa, S. S.; Vera-Nuñez, J. A.; Aguirre-Medina, J. F. y Peña-Cabriales, J. J. 2011. Efecto de los biofertilizantes sobre la asimilación de nitrógeno por el cultivo de trigo. Rev. Mex. Cienc. Agríc. 9(2):281-289. https://doi.org/10.29312/ remexca.v9i2.1071.

Grageda-Cabrera, O. A.; Díaz-Franco, A.; Peña-Cabriales, J. y Vera-Nuñez, J. 2012. Impacto de los biofertilizantes en la agricultura. Rev. Mex. Cienc. Agríc. 3(6):1261-1274.

Harwood, J. 2009. Peasant friendly plant breeding and the early years of the Green Revolution in Mexico. Agricultural History Society. 83(3):384-410. http://doi.org/10.3098/ah. 2009.83.3.384.

Hermosa, R.; Viterbo, A.; Chet, I. and Monte, E. 2012. Plant-beneficial effects of Trichoderma and of its genes. Microbiol. Res. 158(1):17-25. http://doi.org/10.1099/mic.0.052274-0.

Hindersah, R.; Setiawati, M.; Asmiran, P. and Fitriatin, B. 2020. Formulation of Bacillus and Azotobacter, Consortia in liquid cultures: preliminary research on microbes-coated urea. Inter. J. Agric. System. IJAS. 8(1):1-10. http://doi.org/10.20956/ijas.v8i1.2283.

Hipolito-Romero, E.; Carcaño-Montiel, M. G.; Ramos-Prado, J. M.; Vazquez-Cabañas, E. A.; López-Reyes, L. y Ricaño-Rodríguez, J. 2017. Efecto de inoculantes edáficos mixtos en el desarrollo temprano de cultivares mejorados de cacao (Theobroma cacao L.) en un sistema agroforestal tradicional del norte de Oaxaca, México. Rev. Argentina Microbiol. 49(4):356365. https://doi.org/10.1016/j.ram.2017.04.003. 
Huerta-Sobalvarro, K. y Martínez-Centeno, A. 2018. La revolución verde. Revista Iberoamericana de Bioeconomía y Cambio Climático 4(8):1040-1052. http://dx.doi.org/10.5377 /ribcc.v4i8.6717.

Jacoby, R.; Peukert, M.; Succurro, A.; Koprivova, A. and Kopriva, S. 2017. The role of soil microorganisms in plant mineral nutrition -current knowledge and future directions. Frontiers in Plant Science 8:1-19. http://doi.org/10.3389/fpls.2017.01617.

Jha, P. N.; Gomaa, A.; Yanni, Y. G.; El-Saadany, A. E.; Stedtfeld, T. M.; Stedtfeld, R. D.; Gantner, S.; Chai, B.; Cole, J.; Hashsham, S. A and Dazzo, F. 2020. Alterations in the endophyteenriched root-associated microbiome of rice receiving growth-promoting treatments of urea fertilizer and Rhizobium biofertilizer. Microbial Ecology. 79(2):367-382. http://doi.org/10.1007/s00248-019-01406-7.

Jiménez-Gómez, A.; García-Estévez, I.; García-Fraile, P.; Escribano-Bailón, M. T. and Rivas, R. 2020. Increase in phenolic compounds of Coriandrum sativum L. after the application of a Bacillus halotolerans biofertilizer. J. Sci. Food Agric. 100(6):2742-2749. http://doi.org/10.1002/jsfa.10306.

Khalid, A; Arshad, M. and Zahir, Z. A. 2004. Screening plant growth-promoting rhizobacteria for improving growth and yield of wheat. J. Appl. Microbiol. 96(3):473-480. http://doi.org/10.1046/j.1365-2672.2003.02161.x.

Khashei, S.; Etemadifar, Z. and Rahmani, H. R. 2020. Multifunctional biofertilizer from Pseudomonas putida PT: A potential approach for simultaneous improving maize growth and bioremediation of cadmium-polluted soils. Biological J. Microorganism. 8(32):117-129.

Kiuk, Y.; Rai, I. N. and Kesumadewi, A. A. I. 2019. The effectiveness of indigenous Endomycorrhiza and Rhizobium inoculum in increasing nutrient uptake and yield of soybean in dry land. Inter. J. Biosci. Biotechnol. 7(1):18-30. https://doi.org/ 10.24843/IJBB.2019.v07.i01.p03.

Kumar, A.; Bahadur, I.; Maurya, B. R.; Raghuwanshi, R.; Meena, V. S.; Singh, D. K. and Dixit, J. 2015. Does a plant growth-promoting rhizobacteria enhance agricultural sustainability? J. Pure Appl. Microbiol. 9(1):715-724.

Lami, M. J.; Adler, C.; Caram-Di Santo, M. C.; Zenoff, A. M.; de Cristóbal, R. E.; Espinosa-Urgel, M. and Vincent, P. A. 2020. Pseudomonas stutzeri MJL19, a rhizosphere-colonizing bacterium that promotes plant growth under saline stress. J. Appl. Microbiol. https://doi.org/10.1111/jam.14692.

Lekatompessy, S.; Murniati, E.; Kartika, T. and Sukiman, H. I. 2020. Study of the presence of Rhizobium bacteria which were inserted into the cell tissue of soybean seed using vacuum technology. Earth Environ. Sci. 439(1):012014. doi:10.1088/1755-1315/439/1/012014.

Lobell, D. B.; Ortiz-Monasterio, J. I.; Asner, G. P.; Matson, P. A.; Naylor, R. L. and Falcon, W. P. 2005. Analysis of wheat yield and climatic trends in Mexico. Field Crops Res. 94(15):250256. https://doi.org/10.1016/j.fcr.2005.01.007.

López-Feldman, A. J. y Hernández-Cortés, D. 2016. Cambio climático y agricultura, una revisión de la literatura con énfasis en Ámerica Latina. El trimestre económico. 83(332):459-496. http://dx.doi.org/10.20430/ete.v83i332.231.

Lugtenberg, B. J. J. and Dekkers, L. C. 1999. What makes Pseudomonas bacteria rhizosphere competent? Envirom. Microbiol. 1(1):9-13. https://doi.org/10.1046/j.14622920.1999.00005.x. 
Mandal, A.; Sarkar, B.; Mandal, S.; Vithanage, M.; Patra, A. K. and Manna, M. C. 2020. Impact of agrochemicals on soil health. In: agrochemicalss detection, treatment and remediation. (Ed.). Kuhl, M. and Butterworth-Hinemann, L. (Ed.). Oxford. 161-187. https://doi.org/ 10.1016/B978-0-08-103017-2.00007-6.

McCullough, E. and Matson, P. 2016. Evolution of the knowledge system for agricultural development in the Yaqui Valley, Sonora, Mexico. Proceedings of the National Academy of Sciences of the United States of America. 113(17):4609-4614. http://doi.org/10.1073/ pnas. 1011602108.

Metwally, R. A. and Al-Amri, S. M. 2020. Individual and interactive role of Trichoderma viride and arbuscular mycorrhizal fungi on growth and pigment content of onion plants. Letters Appl. Microbiol. 70(2): 541-556. http://doi.org/10.1111/lam.13246.

Moreno-Reséndez, A.; Carda-Mendoza, V.; Reyes Carrillo, J. L.; Vásquez Arroyo, J. y Cano Ríos, P. 2018. Rizobacterias promotoras del crecimiento vegetal: una alternativa de biofertilización para la agricultura sustentable. Rev. Colombiana Biotecnol. 20(1):68-83. https://doi.org/10.15446/rev.colomb.biote.v20n1.73707.

Mota, I.; Sánchez-Sánchez, J.; Pedro, L. G. and Sousa, M. J. 2020. Composition variation of the essential oil from Ocimum basilicum L. cv. Genovese Gigante in response to Glomus intraradices and mild water stress at different stages of growth. Biochem. Systematics Ecol. 90:104021. https://doi.org/10.1016/j.bse.2020.104021.

Nadeem, S. M.; Ahmad, M.; Zahir, Z. A.; Javaid, A. and Ashraf, M. 2014. The role of mycorrhizae and plant growth promoting rhizobacteria (PGPR) in improving crop productivity under stressful environments. Biotechnol. Advances. 32(2):429-448. https://doi.org/10.1016/ j.biotechadv.2013.12.005.

Naylor, R. L.; Falcon, W. P. and Puente-González, A. 2001. Policy reforms and Mexican agriculture: views from the Yaqui Valley (Centro Internacional de Mejoramiento de Maíz y Trigo, Mexico City, Mexico), Economics Program Paper. No. 01-01.

Parra-Cota, F. I.; Peña-Cabriales, J. J.; de los Santos-Villalobos, S.; Martínez-Gallardo, N. A. and Délano-Frier, J. P. 2014. Burkholderia ambifaria and B. caribensis promote growth and increase yield in grain amaranth (Amaranthus cruentus and A. hypochondriacus) by improving plant nitrogen uptake. PLoS ONE 9(2):e88094. https://doi.org/10.1371/journal.pone.0088094.

Passricha, N.; Saifi, S. K.; Sikka, V. K.; Tuteja, N. and Shah, I. 2020. Multilegume biofertilizer: a dream. Molecular Aspects of Plant Beneficial Microbes in Agriculture. 35-45 pp. https://doi.org/10.1016/B978-0-12-818469-1.00003-1.

Reyes-Ortiz, G. E. y Cortés-Sánchez, J. D. 2017. Intensidad en el uso de fertilizantes en América Latina y el Caribe (2006-2012). Bioagro. 29(1):45-52.

Rojas-Padilla, J.; Chaparro-Encinas, L. A.; Robles-Montoya, R. I. and de los Santos Villalobos, S. 2020. Growth promotion on wheat (Triticum turgidum L. subsp. durum) by co-inoculation of native Bacillus strains isolated from the Yaqui Valley, Mexico. Nova Scientia. 12(1):127. doi.org/10.21640/ns.v12i24.2136.

Saharan, B. S. and Nehra, V. 2011. Plant growth promoting rhizobacteria: a critical review. Life Sci. Medicine Res. 21(1):1-30.

Sanjuán-Pinilla, J. y Moreno-Sarmiento, N. 2010. Aplicación de insumos biológicos: una oportunidad para la agricultura sostenible y amigable con el medioambiente. Rev. Colombiana Biotecnol. 12(1):4-7. 
Santoyo, G.; Sanchez-Yañez, J. and de los Santos-Villalobos, S. 2019. Methods for detecting biocontrol and plant growth-promoting traits in Rhizobacteria. Mexico. https://doi.org/ 10.1007/978-981-13-5767-1_8.

Snyder, C. S. 2009. Eficiencia de uso del nitrógeno. Desafíos mundiales, tendencias futuras. Informaciones Agronómicas. 75(1):1-6.

Spolaor, L. T.; Gonçalves, L. S. A.; Santos, O.; Martínez-Oliveira, A. L.; Scapim, C. A.; BengosiBertagna, F. A. and Kuki, M. C. 2016. Plant growth-promoting bacteria associated with nitrogen fertilization at topdressing in popcorn agronomic performance. Bragantia. 75(1):33-40. http://dx.doi.org/10.1590/1678-4499.330.

Tahir, M.; Naeem, M. A.; Shahid, M.; Khalid, U.; Farooq, A. B. U.; Ahmad, N.; Ahmad, I.; Arshad, M. and Waqar, A. 2020. Inoculation of pqq E gene inhabiting Pantoea and Pseudomonas strains improves the growth and grain yield of wheat with a reduced amount of chemical fertilizer. Journal of Applied Microbiology. https://doi.org/10.1111/jam.14630.

Trujillo-Roldan, M. A.; Valdez-Cruz, N. A.; Gonzalez-Monterrubio, C. F.; Acevedo-Sánchez, E. V.; Martínez-Salinas, C.; García-Cabrera, R. I.; Gamboa-Susnavart, R. A.; Marín-Palacio, L. D.; Villegas, J. and Blancas-Cabrera, A. 2013. Scale-up from shake flask to pilot-scale production of the plant growth-promoting bacterium Azospirillum brasilense for preparing a liquid inoculant formulation. Appl. Microbiol. Biotechnol. 97(22):9665-9674. Doi: 10.1007/s00253-013-5199-9.

Valenzuela-Aragon, B.; Parra-Cota, F. I.; Santoyo, G.; Arellano-Wattenbarger, G. L. y de los Santos-Villalobos S. 2019. Plant-assisted selection: a promising alternative for in vivo identification of wheat (Triticum turgidum L. subsp. durum) growth promoting bacteria. Plant Soil. 435(1):367-384. https://doi.org/10.1007/s11104-018-03901-1.

Villa-Rodríguez, E.; Parra-Cota, F.; Castro-Longoria, E.; López-Cervantes, J. and de los SantosVillalobos, S. 2019. Bacillus subtilis TE3: a promising biological control agent against Bipolaris sorokiniana, the causal agent of spot blotch in wheat (Triticum turgidum L. subsp. durum). Biological Control. 132:135-143. https://doi.org/10.1016/j.biocontrol. 2019.02.012.

Villarreal-Delgado, M. F.; Villa-Rodríguez, E. D.; Cira-Chávez, L. A.; Estrada-Alvarado, M. I.; Parra-Cota, F. I. and De los Santos-Villalobos, S. 2018. The genus Bacillus as a biological control agent and its implications in agricultural biosecurity. Mexican J. Phytopathol. 36(1):95-130. https://doi.org/10.18781/R.MEX.FIT.1706-5.

Wang, M.; Bian, Z.; Shi, J.; Wu, Y.; Yu, X.; Yang, Y.; Ni, H.; Chen, H.; Bian, X.; Li, T.; Zhang, Y.; Jiang, L. and Tu, Q. 2020. Effect of the nitrogen-fixing bacterium Pseudomonas protegens $\mathrm{CHA} 0-\Delta$ retS-nif on garlic growth under different field conditions. Industrial Crops and Products. 145:111982. https://doi.org/10.1016/j.indcrop.2019.111982.

WRM. 2004. World Rainforest Movement. La colonización y papel de la agricultura en pocas palabras. Boletín 85 .

Zhang, F.; Dou, K.; Liu, C.; Chen, F.; Wu, W.; Yang, T.; Li, L.; Liu, T. and Yu, L. 2020. The application potential of Trichoderma T-soybean containing 1-aminocyclopropane-1carboxylate for maize production. Physiol. Mol. Plant Pathol. 110:101475. https://doi.org/10.1016/j.pmpp.2020.101475. 